\title{
Variations of Intracranial Dural Venous Sinus Diameters from Birth to 20 Years of Age: An MRV-Based Study
}

\author{
(D) A.S. Larson, (D) G. Lanzino, and (D)W. Brinjikji
}

\begin{abstract}
BACKGROUND AND PURPOSE: The role of the dural venous sinus system in cerebrovascular pathology and the understanding of normal developmental patterns and sizes of the dural venous sinus system continue to expand. The purpose of this study was to review MR venograms to elucidate developmental patterns and diameters of the major dural venous sinuses from 0 to 20 years of age.
\end{abstract}

MATERIALS AND METHODS: All available MR venograms of patients 0-20 years of age who presented to our institution were retrospectively reviewed. Patient age at the time of image acquisition was noted, and measurements were taken of the diameters of the major dural venous sinuses. The presence of embryonic sinuses including the persistent falcine sinus and the occipital sinus was noted. Dominance patterns of the transverse sinus system were determined. Mean diameters of each sinus were plotted as a function of age. The prevalence of persistent prenatal sinuses and transverse sinus-dominance patterns was compared across ages.

RESULTS: A total of 429 MR venograms from 429 patients were reviewed. All dural venous sinuses demonstrated a maximal growth rate from 0 to 7 years of age and reached maximal diameters around 5-10 years of age. The prevalence of falcine sinuses and occipital sinuses trended downward across increasing age categories $(P=.09$ and, $<.0001$, respectively).

CONCLUSIONS: Dural venous sinuses demonstrate maximal growth between 0 and 7 years of age and reach adult size around 510 years of age. Involution of the prenatal sinuses continues to take place after birth into childhood but is largely absent in early adulthood.

ABBREVIATIONS: DVS = dural venous sinus; SSS = superior sagittal sinus; VOG $=$ vein of Galen

E vidence continues to accumulate supporting the idea that the dural venous sinus (DVS) system is a plastic, active player in cerebrovascular pathology rather than a fixed and immutable entity. ${ }^{1,2}$ As the role that the DVS system plays in cerebrovascular disease continues to expand, an understanding of the normal developmental patterns of the DVS system becomes increasingly important. The fixed anatomy of the DVS system and the prevalence of certain anatomic variations are relatively well-understood. ${ }^{3,4}$ The developmental patterns of individual components of the DVS system from birth into adulthood, however, remain relatively unknown. This study consisted of the following 4 objectives: 1) to

Received May 24, 2020; accepted after revision August 5.

From the Departments of Radiology (A.S.L., G.L., W.B.) and Neurosurgery (G.L., W.B.), Mayo Clinic, Rochester, Minnesota.

Please address correspondence to Anthony Larson, BS, Department of Radiology, Mayo Clinic, 200 First St SW, Rochester, MN 55905; e-mail: lars4689@umn.edu; @TonyLarsonBS

Indicates article with supplemental on-line table.

http://dx.doi.org/10.3174/ajnr.A6816 elucidate the growth patterns of each dural venous sinus from birth to 20 years of age, 2) to compare the mean size of each dural venous sinus among ages, 3 ) to compare the prevalence of persistent prenatal sinuses among ages, and 4) to determine the prevalence of transverse sinus-dominance patterns among ages.

\section{MATERIALS AND METHODS \\ Patients}

This was a cross-sectional retrospective study of MRVs obtained at a single institution. Institutional review board approval was obtained before the initiation of this study. All included patients provided written informed consent for involvement in research activities at our institution. MR venography was chosen as the imaging technique over CT venography because younger populations at our institution preferentially undergo MRV over CTV to mitigate radiation exposure; therefore, a larger population of patients 20 years of age and younger had available MRV imaging studies. MR venogram studies of the cerebral venous system that were obtained between 2017 and 2019 were reviewed. These 


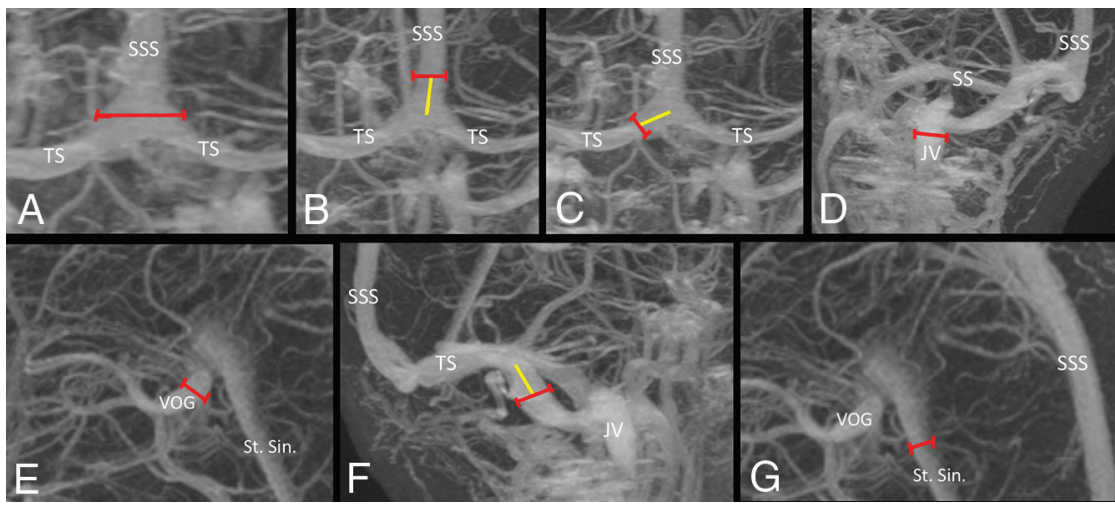

FIG 1. Measurement strategy for determining the diameter of components of dural venous sinuses. $A$, The maximal diameter of torcula is obtained. Superior sagittal sinus $(B)$ and bilateral transverse sinuses $(C)$ are measured (red line) $1 \mathrm{~cm}$ away from the center of the torcula (yellow line). $D$, The maximal diameter of the internal jugular vein is measured at the skull base. $E$, The maximal diameter of the vein of Galen is obtained. F. The sigmoid sinuses are measured (red line) $1 \mathrm{~cm}$ distal to the transverse sigmoid junction (yellow line). G, The maximal diameter of the straight sinus is obtained. JV indicates jugular vein; SS, sigmoid sinus; St. Sin., straight sinus; TS, transverse sinus; SSS, superior sagittal sinus; VOG, vein of Galen.

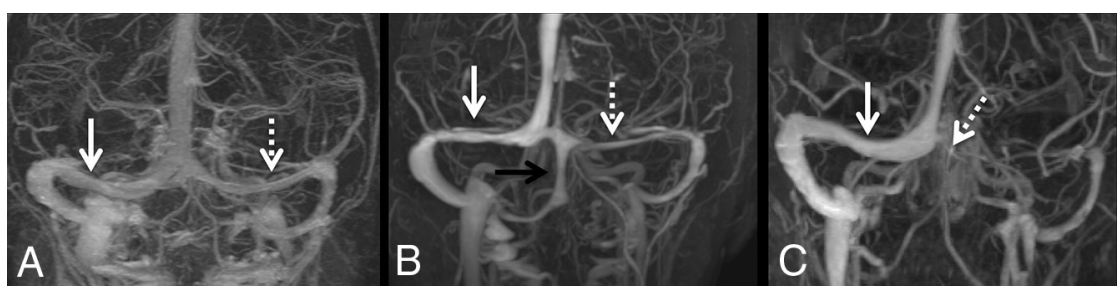

FIG 2. Examples of various transverse venous sinus anatomy patterns. $A$, Codominance between the right (solid arrow) and left (dashed arrow) transverse sinuses. B, Right-side-dominant (solid white arrow) transverse sinus compared with the left (dashed white arrow), with an occipital sinus noted (black arrow). C, Left-side-atretic transverse sinus (dashed arrow) with flow through the right (solid white arrow).

included both contrast-enhanced and non-contrast-enhanced venograms. The indications for obtaining an MRV included headache, migraine, ruling out venous sinus thrombosis, seizures, coagulation defects, papilledema, postconcussive syndrome, or a history of malignancy. In patients with multiple MR venograms, only the earliest study was included. Studies were excluded if the patient was found to have 1 of following vascular anomalies: an arteriovenous malformation involving a draining vein into a dural venous sinus, a dural arteriovenous fistula, dural venous sinus thrombosis, vein of Galen (VOG) malformation, or any kind of external compression of the venous sinuses. Data extracted from each MRV study included the diameter of the specific components of the DVS system, the presence of any persistent prenatal sinus including the persistent falcine sinus or occipital sinus, and the dominance patterns of the transverse venous system. Patient age at the time of MRV imaging was obtained. We used the following age categories: younger than $1,1-5,6-10,11-15$, and 16-20 years of age.

\section{Venogram Parameters}

Gadolinium bolus MRV examinations were performed on multiple 3T scanners (Discovery M750; GE Healthcare) throughout our institution using a 64-channel coil. Images were obtained in the sagittal plane. We used the following scan parameters: $\mathrm{TR}=3.83 \mathrm{~ms}$, $\mathrm{TE}=1.39 \mathrm{~ms}$, section thickness = $0.65 \mathrm{~mm}$, skip $=20 \%, \mathrm{FOV}=240 \mathrm{~mm}$.

\section{Measurements of the Dural Venous Sinus System}

All measurements were made by a single author. All measurements were obtained on a 3D MR venogram with or without contrast. Measurements were obtained of the diameter of the following DVS components: superior sagittal sinus (SSS), straight sinus, VOG, torcular Heterophili, bilateral transverse sinuses, bilateral sigmoid sinuses, and bilateral internal jugular veins. The SSS and transverse sinuses were measured $1 \mathrm{~cm}$ from the middle of the torcula as performed previously. ${ }^{5}$ The maximum diameters of the straight sinus, VOG, and torcula were measured. Because the diameters of the straight sinus and VOG could be relatively small in some cases (particularly in younger patients), the maximum diameter of each was measured on each study for consistency and ease of measurement across studies. The diameters of the sigmoid sinuses were measured $1 \mathrm{~cm}$ distal to the transverse-sigmoid junction, and the diameter of the internal jugular vein was measured at the base of the skull. ${ }^{5}$

The anatomy of the transverse sinus system was classified as codominant, right-side-dominant, left-side-dominant, right-side-atretic, or leftside-atretic. All measurements were performed in QREADS imaging software (Mayo Clinic). Figure 1 is an example of the measurement process, and Fig 2 shows sample patterns of transverse sinus anatomy.

\section{Validation of Venous Sinus Measurements}

To validate measurements made on $3 \mathrm{D}$ MR venograms, we selected 20 patients with available $2 \mathrm{D}$ MR venograms and obtained measurements of each DVS component measured on 3D images. Patients were selected from each age category to represent the age distributions of the overall population included in the study. The SSS was measured in the axial plane approximately $1 \mathrm{~cm}$ from the torcula. The torcula and internal jugular veins were, likewise, measured in the axial plane. The largest dimensions of the straight sinus and VOG were measured in the coronal plane. The bilateral transverse sinuses were measured in the sagittal plane $1 \mathrm{~cm}$ from the torcula. The sigmoid sinuses were measured in the axial plane approximately $1 \mathrm{~cm}$ from the transverse-sigmoid junction. Mean values of each DVS component as measured on 2D MR venograms were calculated and compared with the mean values as measured on the 3D MR venograms of the 20 patients included. 


\section{Statistical Analysis}

Means and SDs were calculated for continuous variables. Student's 2-tailed $t$ test was used to determine significance between mean diameter values in the imaging-validation analysis. Percentages were calculated for binary/categoric variables. The $\chi^{2}$ test was used to determine the significance among categoric variables. To compare the diameters of individual venous sinuses by age, we plotted measurements against age and applied a trivariate fit to the graph. Any $P$ value $<.05$ was considered statistically significant. All calculations took place in Excel (Microsoft) and SPSS Statistics for Windows (Version 25.0; IBM).

\section{RESULTS}

\section{Patients and Baseline Information}

In total, 488 venograms from 477 patients were reviewed. Three patients had $3 \mathrm{MR}$ venograms included, and 5 patients had 2 venograms included. Fifty-five imaging studies were excluded for

\section{Table 1: Baseline demographics}

\begin{tabular}{lc}
\hline \multicolumn{1}{c}{ Demographics } \\
\hline Total MR venograms Included \\
Total patients included & 429 \\
No. of MR venograms with IV contrast (\%) & 429 \\
Age categories (No.) (\%) (yr) & $327(76.2)$ \\
0 & \\
$1-5$ & $25(5.8)$ \\
$6-10$ & $24(5.6)$ \\
$11-15$ & $21(4.9)$ \\
$16-20$ & $70(16.3)$ \\
Mean age (SD) (yr) & $289(67.4)$ \\
Male (No.) (\%) & $15.1(5.9)$ \\
\hline
\end{tabular}

the following reasons: AVM draining into a dural venous sinus $(n=6)$, venous sinus compression $(n=1)$, dural arteriovenous fistula $(n=3)$, venous sinus thrombosis $(n=43)$, and VOG malformation $(n=2)$. Three patients had multiple venograms: Two patients had 2 venograms, and 1 patient had 3. After exclusion, we included 429 venograms from 429 patients. Patient and baseline information are further summarized in Table 1.

\section{Venous Sinus Diameter by Age}

The following data are summarized in Table 2. Regarding the transverse sinuses, the largest mean dimension for the right transverse sinus was found in the 6- to 10-year age category $(8.1 \pm 2.0 \mathrm{~mm})$, whereas the largest mean dimension for the left transverse sinus was found in 6-10 and 11-15 age categories $(6.4 \pm 2.5 \mathrm{~mm}$ and $6.4 \pm 2.4 \mathrm{~mm}$, respectively). The torcula ranged from $8.4 \pm 4.1 \mathrm{~mm}$ in the younger than 1-year category to $17.4 \pm 4.1 \mathrm{~mm}$ in the $16-20$ age category. The SSS ranged from $4.0 \pm 1.5 \mathrm{~mm}$ in the younger than 1-year category to $8.8 \pm 1.6 \mathrm{~mm}$ in the 6 - to 10 -year category. The straight sinus ranged from $3.9 \pm 1.1 \mathrm{~mm}$ in the younger than 1-year category to $5.4 \pm 2.7 \mathrm{~mm}$ in the 1 - to 5 -year age category. The VOG ranged from $3.2 \pm 0.8 \mathrm{~mm}$ in younger than 1-year category to $4.9 \pm 1.0 \mathrm{~mm}$ in the 11- to 15-year age category. Regarding the right and left sigmoid sinuses, the maximum size was observed in the $11-15$ category $(10.1 \pm 2.4 \mathrm{~mm})$ and the 16-20 age category $(9.0 \pm 2.1 \mathrm{~mm})$, respectively. Regarding the internal jugular veins, the maximum mean diameter was observed in the 16-20 age category on both right and left sides $(10.0 \pm 2.2$ and $8.1 \pm 2.1 \mathrm{~mm}$, respectively).

To determine at which specific age the sinuses of the DVS system reached maximal diameter, we plotted the diameter of

$\underline{\text { Table 2: Variations in size measurements of cerebral venous system components by age }{ }^{\mathrm{a}}}$

\begin{tabular}{lccccc}
\hline & \multicolumn{5}{c}{ Age Category (Years) } \\
\cline { 2 - 6 } & $<1$ & $1-5$ & $6-10$ & $11-15$ & $16-20$ \\
\hline No. & 25 & 24 & 21 & 70 & 289 \\
Right transverse sinus diameter $(\mathrm{mm})$ & $3.7(2.1)$ & $5.4(2.8)$ & $8.1(2.0)$ & $7.1(2.2)$ & $7.6(2.1)$ \\
Left transverse sinus diameter $(\mathrm{mm})$ & $3.1(2.2)$ & $5.9(3.1)$ & $6.4(2.5)$ & $6.4(2.4)$ & $6.1(2.0)$ \\
Torcula & $8.4(4.1)$ & $13.3(4.5)$ & $15.4(3.4)$ & $16.5(4.1)$ & $17.4(4.1)$ \\
Superior sagittal sinus & $4.0(1.5)$ & $7.4(2.5)$ & $8.8(1.6)$ & $8.6(1.9)$ & $8.7(1.6)$ \\
Straight sinus & $3.9(1.1)$ & $5.4(2.7)$ & $5.2(1.6)$ & $5.2(1.5)$ & $4.8(1.3)$ \\
Vein of Galen & $3.2(0.8)$ & $4.4(1.1)$ & $4.8(0.9)$ & $4.9(1.0)$ & $4.8(1.0)$ \\
Right sigmoid sinus & $5.0(2.1)$ & $6.7(1.4)$ & $8.6(2.4)$ & $10.1(2.4)$ & $10.0(1.9)$ \\
Left sigmoid sinus & $4.0(2.0)$ & $7.3(2.8)$ & $8.0(2.2)$ & $8.8(2.5)$ & $9.0(2.1)$ \\
Right internal jugular vein & $4.4(1.5)$ & $6.8(1.4)$ & $9.0(2.2)$ & $9.9(2.2)$ & $10.0(2.2)$ \\
Left internal jugular vein & $3.9(1.4)$ & $6.8(2.8)$ & $7.4(1.7)$ & $7.8(2.2)$ & $8.1(2.1)$ \\
\hline
\end{tabular}
each sinus as a function of age. The plots are shown in Figs 3 and 4. The SSS, torcula, straight sinus, and VOG all reached maximal diameter by $7-10$ years of age. Both the right and left transverse venous sinuses reached maximal size around 5-7 years of age. The left sigmoid sinus reached maximal diameter around 7-10 years of age, while the right sigmoid sinus reached maximal size around 12.5 years of age. The right and left internal jugular veins reached maximal size around 710 years of age.
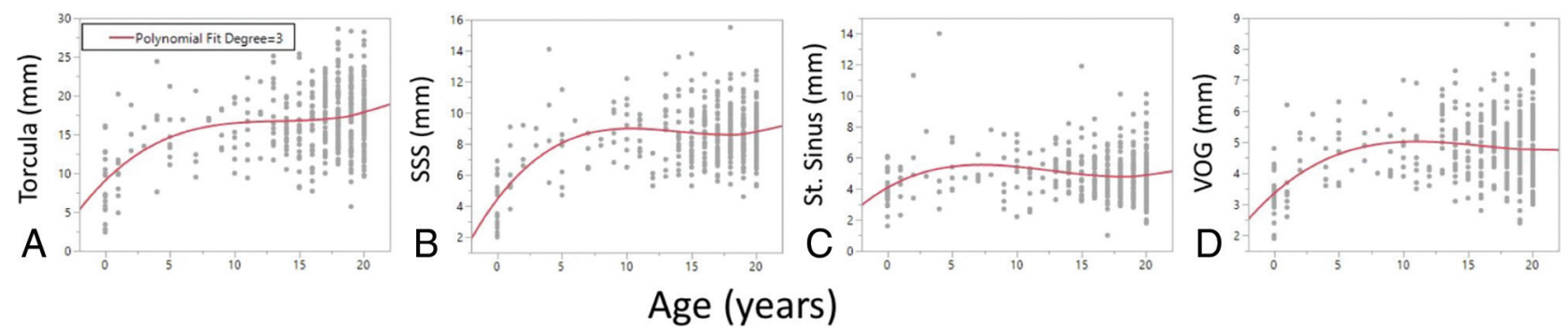

FIG 3. Variations in venous size by age in the torcula $(A)$, superior sagittal sinus $(B)$, straight sinus $(C)$, and vein of Galen $(D)$. SSS indicates superior sagittal sinus; St. Sinus, straight sinus; VOG, vein of Galen. 

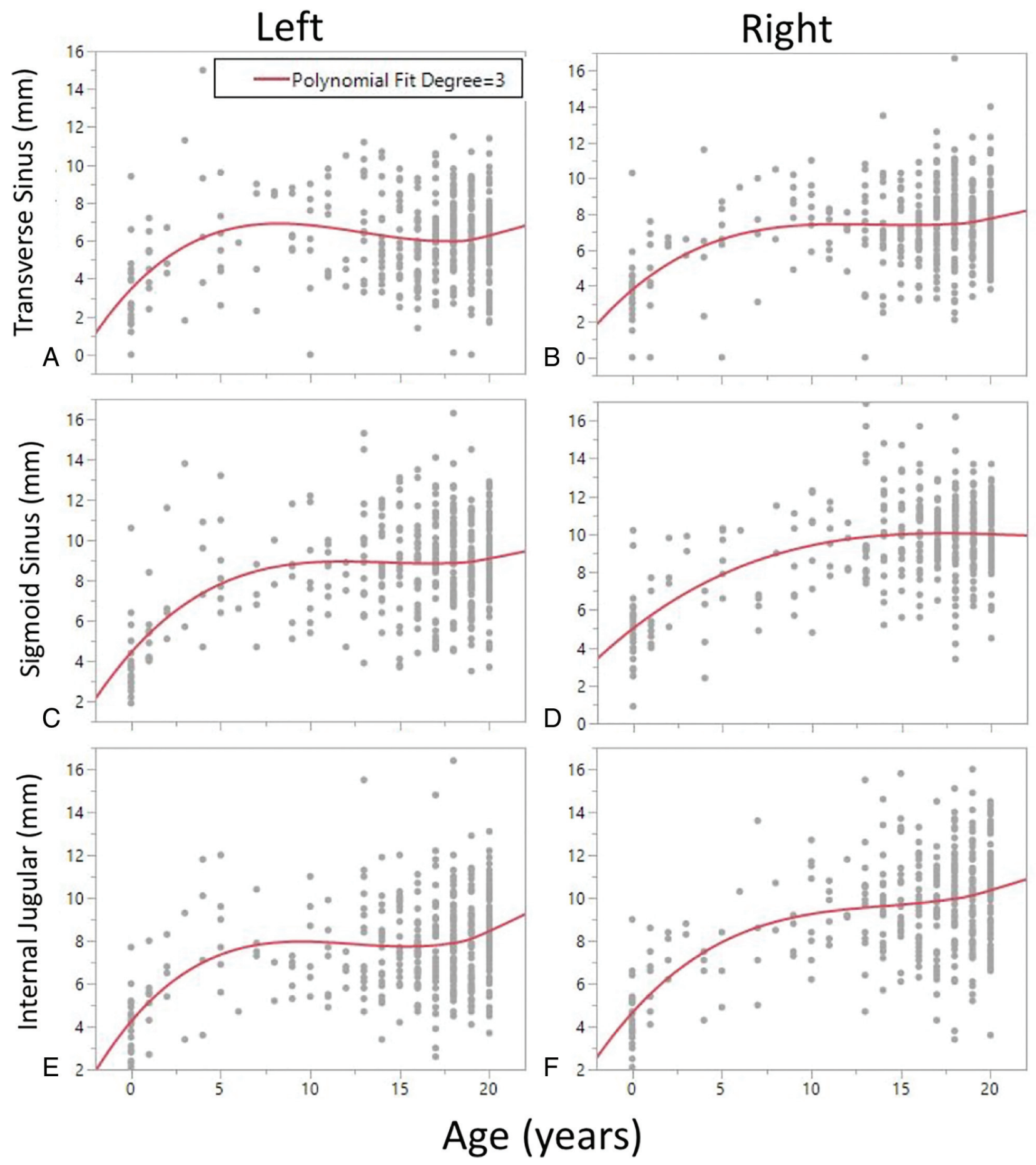

FIG 4. Variations in venous sinus size by age in the left $(A)$ and right $(B)$ transverse venous sinuses, left $(C)$ and right $(D)$ sigmoid sinuses, and left $(E)$ and right $(F)$ internal jugular veins.

\section{Validation of Venous Sinus Measurements}

The age distribution of patients included in the validation analysis was as follows: One patient (5\%) was younger than 1 year of age, 1 patient (5\%) was between 1 and 5 years of age, 1 patient (5\%) was between 6 and 10 years of age, 3 patients (15\%) were between 11 and 15 years of age, and 14 patients (70\%) were between 16 and 20 years of age. For all components of the DVS system, there were no differences between the mean values of the measurements made on 2D MR venograms compared with the mean values of measurements made on 3D MR venograms. These data are summarized in the On-line Table.

\section{Persistent Prenatal Sinuses by Age Category}

The following data are summarized in Table 3. A persistent falcine sinus was observed in $8.0 \%$ of the younger than 1-year age category. This percentage trended downward to $1.4 \%$ of the 16 to 20-year age category, yet the difference between groups did not reach statistical significance $(P=.09)$. A similar downward trend 
Table 3: Persistence of postnatal sinuses by age

\begin{tabular}{lcccccc}
\hline & \multicolumn{7}{c}{ Age Category (Years) } \\
\cline { 2 - 7 } & $<1$ & $\mathbf{1 - 5}$ & $\mathbf{6 - 1 0}$ & $\mathbf{1 1 - 1 5}$ & $\mathbf{1 6 - 2 0}$ & $\boldsymbol{P}$ Value \\
\hline No. & 25 & 24 & 21 & 70 & 289 & - \\
Persistent falcine sinus (No.) (\%) & $2(8.0)$ & $2(8.3)$ & $1(4.8)$ & $2(2.9)$ & $4(1.4)$ & .09 \\
Occipital sinus (No.) (\%) & $6(24.0)$ & $6(25.0)$ & $1(4.8)$ & $6(8.7)$ & $8(2.8)$ & $<.0001$ \\
\hline
\end{tabular}

Note:-- indicates that no $P$-value was calculated.

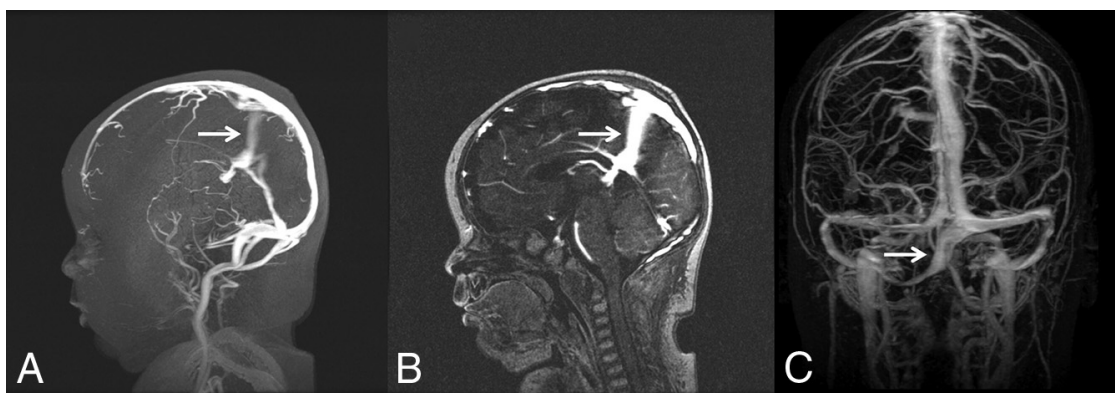

FIG 5. Examples of embryonic sinuses. Persistent falcine sinus in a 2-month-old infant seen on 3D-MRV (A) and a sagittal section of 2D-MRV (B, arrows). C, Occipital sinus (arrow).

Table 4: Transverse venous sinus anatomy patterns by age

\begin{tabular}{|c|c|c|c|c|c|c|}
\hline & \multicolumn{6}{|c|}{ Age Category (Years) } \\
\hline & $<1$ & $1-5$ & 6-10 & $11-15$ & $16-20$ & $\begin{array}{c}P \\
\text { Value } \\
\end{array}$ \\
\hline No. patients in each age category & 25 & 24 & 21 & 70 & 289 & - \\
\hline $\begin{array}{l}\text { Codominant (No. of patients) (\% } \\
\text { of age group) }\end{array}$ & $12(48.0)$ & $12(50.0)$ & $8(38.1)$ & $37(52.9)$ & 177 (61.2) & .14 \\
\hline Right-dominant & $8(32.0)$ & $4(16.7)$ & 9 (42.9) & $22(31.4)$ & $76(26.3)$ & .18 \\
\hline Left-dominant & $3(12.0)$ & $6(25.0)$ & $3(14.3)$ & 9 (12.9) & $23(8.0)$ & \\
\hline Right-atretic & $1(4.0)$ & $1(4.2)$ & $0(0.0)$ & $1(1.4)$ & $3(1.0)$ & .81 \\
\hline Left-atretic & $1(4.0)$ & $1(4.2)$ & $1(4.8)$ & $1(1.4)$ & $10(3.4)$ & \\
\hline
\end{tabular}

Note:- - indicates that no $P$-value was calculated.

was noted with the occipital sinus, which was present in $24.0 \%$ of the younger than 1-year population and trended downward to $2.8 \%$ of the 16 - to 20 -years of age category $(P<.0001)$. A sample MRV of a persistent falcine sinus and occipital sinus is demonstrated in Fig 5.

\section{Transverse Sinus Anatomy Patterns by Age Category}

Regarding the anatomic makeup of the transverse sinus system, codominance was observed most commonly in all age categories except the 6- to 10-year category, in which right-sided transverse sinus dominance was most commonly observed. Right-sided dominance was more commonly seen than left-sided dominance in all age categories except ages 1-5. There was no difference in the prevalence of codominance across age categories $(P=.14)$. There was no difference in the prevalence of left-versus-rightsided dominance across age categories $(P=.18)$. This was also true when comparing left-versus-right-sided atresia $(P=.81)$. These data are summarized in Table 4.

\section{DISCUSSION}

Our study of 429 MR venograms demonstrates the patterns of development of the major sinuses of the DVS system from birth to
20 years of age. Three important findings are evident: First, dural venous sinuses, in general, demonstrated maximal growth between 0 and 7 years of age, and most sinuses reached adult size around 7-10years of age. This finding emphasizes the postnatal plasticity of the DVS system. Second, a higher prevalence of the persistent prenatal sinuses was found in younger age categories compared with older categories, implying that involution of these sinuses continues to take place after birth into childhood but largely ceases in early adulthood. Finally, we found no difference in patterns of transverse venous sinus drainage across age categories, suggesting that transverse venous sinus dominance patterns are maintained into early adulthood.

The main finding from our study is that postnatal growth of the DVS system continues until 10 years of age after which diameters remain stable. These findings track closely with the rates of head circumference growth as well as growth of the intracranial arteries across time. ${ }^{6}$ There are important clinical implications to understanding the rate of change of the cerebral venous system across time. For example, with the growing use of venous sinus stent placement for treatment of idiopathic intracranial hypertension in adults, there have been several reports in the literature describing venous sinus stent placement to treat pediatric patients. ${ }^{7-9}$ An understanding of normal age-related venous sinus diameters would be important for device sizing and treatment planning in these patients. There are also growing reports on mechanical endovascular recanalization of venous sinus thrombosis in the pediatric population, and again, having an understanding of the normal venous sinus diameters would be important for sizing of thrombectomy devices. ${ }^{10,11}$

Our data suggest a gradual involution of the occipital and persistent falcine sinuses from birth to 20 years of age. The occipital sinus originates from the torcular plexus and is discernable as early as the third gestational month. ${ }^{12,13}$ Secondary to increased hemodynamic burden within the developing posterior fossa, the occipital sinus demonstrates a rapid expansion in the fourth and fifth gestational months, followed by diminution in the sixth and seventh months. ${ }^{12,13}$ The presence of an occipital sinus in postnatal life is, therefore, less commonly observed. Similar to the pattern observed in the current study, Widjaja and Griffiths ${ }^{5}$ found an occipital sinus in 9 of 50 patients (18\%), most of whom were younger than of 2 years of age, and none were found in those older than 9 years of age. Additionally, Mizutani et $\mathrm{al}^{14}$ found that 6 of 13 infants (46.2\%) had an occipital sinus, compared with 
only 3 of 35 adults (8.6\%), suggesting that persistent occipital sinuses largely regress by adulthood.

Persistent falcine sinuses are exceptionally rare and are typically present in the context of absent, hypoplastic, or occluded venous outflow tracts. ${ }^{15,16}$ This observation implies that alterations in cerebral venous flow dynamics may result in recanalization of the falcine sinus. In the current study, we observed a trend in which the prevalence of the persistent falcine sinus decreased across age categories; this trend may suggest that venous outflow dynamics normalize with increasing age, thereby prompting involution of the falcine sinus. One separate study found no difference in the prevalence of persistent falcine sinuses between infants and adults. ${ }^{14}$ This finding is likely due to small sample sizes, however, given that persistent falcine sinuses have an exceptionally low prevalence overall. The findings of the current study relating to the prevalence of the falcine and occipital sinuses demonstrate the dynamic nature of the DVS system in younger age categories.

Prenatal formation of the transverse venous sinuses is characterized by rapid expansion in the fourth fetal month, which is followed by contraction to a relatively stable diameter at 7-8 months of fetal life. ${ }^{12,13,17}$ Much variation exists within the anatomic patterns of the transverse venous system. Although many individuals have a codominant transverse venous system, a large proportion of the population exhibits unilateral dominance, typically on the right, suggesting that a single transverse sinus is sufficient in providing outflow for the superficial venous system. ${ }^{13,18}$ Nevertheless, changes in transverse sinus dimensions to compensate for altered flow mechanics may occur. ${ }^{19}$ Our data here demonstrate that transverse sinus patterns do not change as a result of increasing age, suggesting that the in utero establishment of a transverse sinus-dominance pattern is sufficient for maintaining adequate venous drainage from birth into adulthood. Our study may be limited in the conclusions that may be drawn in this regard; however, the transverse sinuses were found to be codominant in every age group other than the 6- to 10 -year-old patients, suggesting that the dural venous sinuses are still developing in younger children and that 1 transverse sinus becomes unilaterally dominant by $6-10$ years of age. If this scenario were the case, however, this pattern of transverse sinus dominance would be prevalent in adulthood as well. Given that this pattern was not found to be prevalent, this issue suggests a potential sampling error in our study. Further study is, therefore, warranted to more fully elucidate developmental patterns of the transverse sinus system.

Fetal development of the cerebral DVS system is highly complex and begins early in fetal development. ${ }^{5,13}$ The findings from our study highlight the incredibly dynamic nature of the dural venous sinus system with rapid growth of some sinuses and regression of others in postnatal life and into early childhood. The dynamic character of the cerebral venous system and its role in the pathogenesis of various intracranial vascular malformations has only recently been acknowledged. ${ }^{2}$ For example, several reports of de novo formation of vascular malformations with venous components imply active pathogenetic involvement of the venous system. ${ }^{20-22}$ Our understanding of the role of the venous system in various pathologic states continues to evolve.

Important limitations of this report must be considered. Our study population was heavily skewed toward those 16-20 years of age; therefore, the prevalence of embryonic sinuses may be underestimated in the younger age categories. Our study is prone to selection bias for 2 reasons: All those who had MRV studies had a clinical indication for imaging, and the patient population was obtained from a single quaternary referral center.

Most important, a minority of studies ( $n=23.8)$ were not performed with contrast, possibly resulting in suboptimal measurements. Our measurements were made on 3D MR venograms, which may make it difficult to ascertain the true diameter of a $2 \mathrm{D}$ structure (ie, the diameter of the lumen of a given venous sinus). Ideally, measurements would be made on a $2 \mathrm{D}$ image on which the image section is perpendicular to the long axis of the vessel. However, our validation analysis found no differences between measurements made on 3D and 2D; therefore, our measurement protocol was sufficient for the purposes of this study. Future studies should adopt stringent quality control measures with regard to the technical aspects of each study because various physiologic and imaging-based parameters may potentially affect the measured diameter of each DVS system component. Future studies should also consist of multiple measurements made by multiple authors for validation purposes.

\section{CONCLUSIONS}

We studied 429 MR venograms to demonstrate patterns of development of the DVS system from birth to 20 years of age. In general, dural venous sinuses demonstrated maximal growth between 0 and 7 years of age, which demonstrates postnatal plasticity of the cerebral venous system. Involution of persistent prenatal sinuses continues to take place after birth into childhood but largely ceases in early adulthood. Transverse venous sinus dominance patterns are likely maintained throughout the aging process.

Disclosures: Giuseppe Lanzino_ UNRELATED: Board Membership: Superior Medical Editing, Nested Knowledge.

\section{REFERENCES}

1. Mullan S, Mojtahedi S, Johnson DL, et al. Embryological basis of some aspects of cerebral vascular fistulas and malformations. J Neurosurg 1996;85:1-8 CrossRef Medline

2. Aboian MS, Daniels DJ, Rammos SK, et al. The putative role of the venous system in the genesis of vascular malformations. Neurosurg Focus 2009;27:E9 CrossRef Medline

3. Bisaria KK. Anatomic variations of venous sinuses in the region of the torcular Herophili. J Neurosurg 1985;62:90-95 CrossRef Medline

4. Cure JK, Van Tassel P, Smith MT. Normal and variant anatomy of the dural venous sinuses. Semin Ultrasound CT MR 1994;15:499-519 CrossRef Medline

5. Widjaja E, Griffiths PD. Intracranial MR venography in children: normal anatomy and variations. AJNR Am J Neuroradiol 2004;25:1557-62 Medline

6. Vaughan J, Taylor M, Leach JL, et al. Abstract TMP113: non-invasive estimate of intracranial arterial luminal diameter in children. Stroke 2020;51(Suppl 1):ATMP113

7. Tibussek D, Distelmaier F, von Kries R, et al. Pseudotumor cerebri in childhood and adolescence: results of a Germany-wide ESPEDsurvey. Klin Padiatr 2013;225:81-85 CrossRef Medline

8. Buell TJ, Starke RM, Ding D, et al. Venous sinus stenting using transcranial access for the treatment of idiopathic intracranial hypertension in a pediatric patient. $J$ Neurosci Rural Pract 2017;8:672-75 CrossRef Medline 
9. Rajpal S, Niemann DB, Turk AS. Transverse venous sinus stent placement as treatment for benign intracranial hypertension in a young male: case report and review of the literature. J Neurosurg 2005;102:342-46 CrossRef Medline

10. Csakanyi Z, Rosdy B, Kollar K, et al. Timely recanalization of lateral sinus thrombosis in children: should we consider hypoplasia of contralateral sinuses in treatment planning? Eur Arch Otorhinolaryngol 2013;270:1991-98 CrossRef Medline

11. Omoto K, Nakagawa I, Park HS, et al. Successful emergent endovascular mechanical thrombectomy for pediatric and young adult cerebral venous sinus thrombosis in coma. World Neurosurg 2019;122:203-08 CrossRef Medline

12. Okudera T, Huang YP, Ohta T, et al. Development of posterior fossa dural sinuses, emissary veins, and jugular bulb: morphological and radiologic study. AJNR Am J Neuroradiol 1994;15:1871-83 Medline

13. Tubbs RS. Anatomy, Imaging and Surgery of the Intracranial Dural Venous Sinuses: E-Book. Elsevier Health Sciences; 2019

14. Mizutani K, Miwa T, Akiyama T, et al. Fate of the three embryonic dural sinuses in infants: the primitive tentorial sinus, occipital sinus, and falcine sinus. Neuroradiology 2018;60:325-33 CrossRef Medline

15. Raybaud CA, Strother CM, Hald JK. Aneurysms of the vein of Galen: embryonic considerations and anatomical features relating to the pathogenesis of the malformation. Neuroradiology 1989;31:109-28 CrossRef Medline
16. Manoj KS, Krishnamoorthy T, Thomas B, et al. An incidental persistent falcine sinus with dominant straight sinus and hypoplastic distal superior sagittal sinus. Pediatr Radiol 2006;36:65-67 CrossRef Medline

17. Kopuz C, Aydin ME, Kale A, et al. The termination of superior sagittal sinus and drainage patterns of the lateral, occipital at confluens sinuum in newborns: clinical and embryological implications. Surg Radiol Anat 2010;32:827-33 CrossRef Medline

18. Durgun B, Ilglt ET, Cizmeli MO, et al. Evaluation by angiography of the lateral dominance of the drainage of the dural venous sinuses. Surg Radiol Anat 1993;15:125-30 CrossRef Medline

19. Nicholson P, Lenck S, Kucharczyk W, et al. Dynamic nature of intracranial venous sinuses in idiopathic intracranial hypertension. Interv Neuroradiol 2020;26:118-20 CrossRef Medline

20. Perrini $P$, Lanzino $G$. The association of venous developmental anomalies and cavernous malformations: pathophysiological, diagnostic, and surgical considerations. Neurosurg Focus 2006;21:e5 CrossRef Medline

21. Campeau NG, Lane JI. De novo development of a lesion with the appearance of a cavernous malformation adjacent to an existing developmental venous anomaly. AJNR Am J Neuroradiol 2005;26:156-59 Medline

22. Brinjikji W, El-Masri AE, Wald JT, et al. Prevalence of cerebral cavernous malformations associated with developmental venous anomalies increases with age. Childs Nerv Syst 2017;33:1539-43 CrossRef Medline 